MATHEMATICS OF COMPUTATION

Volume 70, Number 234, Pages 507-523

S 0025-5718(00)01229-1

Article electronically published on February 23, 2000

\title{
DISCRETE COMPACTNESS AND THE APPROXIMATION OF MAXWELL'S EQUATIONS IN $\mathbb{R}^{3}$
}

\author{
P. MONK AND L. DEMKOWICZ
}

\begin{abstract}
We analyze the use of edge finite element methods to approximate Maxwell's equations in a bounded cavity. Using the theory of collectively compact operators, we prove $h$-convergence for the source and eigenvalue problems. This is the first proof of convergence of the eigenvalue problem for general edge elements, and it extends and unifies the theory for both problems. The convergence results are based on the discrete compactness property of edge element due to Kikuchi. We extend the original work of Kikuchi by proving that edge elements of all orders possess this property.
\end{abstract}

\section{INTRODUCTION}

In the theoretical analysis of finite element methods for Maxwell's equations, as well as in practical applications, we can distinguish two basic model problems. The first is to compute the resonant frequencies or eigenvalues of a bounded perfectly conducting cavity. The second is to compute the electromagnetic field in the cavity due to a known current source (at a nonresonant frequency). If edge finite elements are used to discretize either problem, the first obvious theoretical question is to determine estimates of the convergence rate of the resulting approximation.

For the eigenvalue problem, convergence was proved by Kikuchi [15, 16, 17] using his discrete compactness property. This was only shown to hold for the lowest order edge element space of Nédélec [23] on a tetrahedral mesh. A rate of convergence was not given. A different analysis, due to Levillain [21, unfortunately contains an error in the proof of norm convergence of the appropriate operators.

For the source problem, convergence was first proved by Monk [22] using an extension of Schatz's duality theory for the Helmholtz equation [28]. Unfortunately it was necessary to assume that the cavity is either a convex polyhedron or a smooth domain due to the use of certain a priori estimates in the analysis.

More recently Demkowicz 8 , has observed that convergence for the source problem for the Helmholtz equation is implied by a suitable convergence estimate for the eigenvalue problem. This suggests that both the eigenvalue and source problem should be analyzed using the same theory. Our paper is devoted to analyzing the two problems using the theory of collectively compact operators [2] which requires only pointwise convergence of an appropriate sequence of discrete operators. This approach is standard in the analysis of integral equation methods for time-harmonic

Received by the editor October 27, 1998 and, in revised form, April 1, 1999.

2000 Mathematics Subject Classification. Primary 65N30; Secondary 65N15, 65N25.

Key words and phrases. Finite element methods, discrete compactness, eigenvalues, error estimates. 
wave problems [18] and was used to analyze the approximation of the 2D waveguide problem by Joly et al. in [14]. Indeed our paper has been significantly influenced by that work.

We remark that in [3 4] some mixed approximations to eigenvalue problems for elliptic equations are analyzed by proving the norm convergence of a suitable operator. In particular, in [4], various mixed methods for computing the Dirichlet eigenvalues for Laplace's equations are analysed with the goal of illustrating spurious modes for some standard mixed methods satisfying the Babuška-Brezzi conditions. For other mixed methods, additional assumptions (implying norm convergence of the appropriate operator) are then shown to imply convergence of the eigenvalue problem. This theme is continued in [3]. Our analysis avoids proving norm convergence and offers an alternative approach to problems of this type.

To verify that the theory of collectively compact operators is applicable, we use the discrete compactness property of Kikuchi [17. We extend the proof that this property holds to include edge elements of all orders based on a tetrahedral or hexahedral mesh (under suitable restrictions on the mesh). Our proof is based on recent regularity results due to Costabel and Dauge [7. We restrict ourselves to the $h$-version of the finite element method, but hope to extend our analysis to the $h-p$ version in the future.

Let us now state the two problems we shall analyze. Let $\Omega \subset \mathbb{R}^{3}$ be a bounded, Lipschitz, polyhedral domain with boundary $\Gamma$, and unit outward normal $\boldsymbol{\nu}$. To avoid certain static solutions, we shall assume that $\Gamma$ is connected. For a disconnected boundary $\Gamma$, the theory can be modified in the obvious way. The assumption that $\Omega$ is a Lipschitz domain rules out "screens" and "topological singularities" [7. It would be desirable to extend the theory to include screens which are often used as models of antennas.

The eigenvalue problem is to find an electric field $\boldsymbol{E} \neq 0$ and an electric eigenvalue $\lambda$ such that

$$
\begin{aligned}
\nabla \times \nabla \times \boldsymbol{E} & =\lambda \boldsymbol{E} \text { in } \Omega, \\
\nabla \cdot \boldsymbol{E} & =0 \text { in } \Omega, \\
\boldsymbol{\nu} \times \boldsymbol{E} & =0 \text { on } \Gamma .
\end{aligned}
$$

The assumption that $\Gamma$ is simply connected implies that $\lambda=0$ is not an eigenvalue for this problem. The eigenvalue problem has been studied extensively in the literature (see for example [20]). Under the conditions we have given, there is a discrete set of real eigenvalues $\left\{\lambda_{n}\right\}_{n=1}^{\infty}$ with $0<\lambda_{1} \leq \lambda_{2} \leq \lambda_{3} \cdots$ such that $\lambda_{n} \rightarrow \infty$ as $n \rightarrow \infty$. The eigenspace $W\left(\lambda_{n}\right)$ associated with $\lambda_{n}, n=1, \ldots$, is finite dimensional.

If the wave number $k>0$ of the time harmonic field in $\Omega$ is such that $k^{2}$ is not an electric eigenvalue of $\Omega$, we can also pose the source problem. Given a divergence free current distribution $\boldsymbol{J}$, we seek the electric field $\boldsymbol{E}$ which satisfies

$$
\begin{aligned}
\nabla \times \nabla \times \boldsymbol{E}-k^{2} \boldsymbol{E} & =-\boldsymbol{J} \text { in } \Omega, \\
\nabla \cdot \boldsymbol{E} & =0 \text { in } \Omega, \\
\boldsymbol{\nu} \times \boldsymbol{E} & =0 \text { on } \Gamma .
\end{aligned}
$$

This problem is also well studied (see for example [20]) where existence and uniqueness of a suitable weak solution to this problem is verified for a general divergencefree current source $\boldsymbol{J} \in\left(L^{2}(\Omega)\right)^{3}$. 
The finite element approximation of these problems is based on a suitable variational formulation. We recall that

$$
H_{0}(\operatorname{curl} ; \Omega)=\left\{\boldsymbol{u} \in\left(L^{2}(\Omega)\right)^{3} \mid \nabla \times \boldsymbol{u} \in\left(L^{2}(\Omega)\right)^{3}, \boldsymbol{\nu} \times \boldsymbol{u}=0\right\} .
$$

We denote by $\|\cdot\|$ the $\left(L^{2}(\Omega)\right)^{3}$ (or $L^{2}(\Omega)$ ) norm, and for other Hilbert spaces $H$ we denote by $\|\cdot\|_{H}$ the norm on that space. Finally we denote

$$
(\boldsymbol{u}, \boldsymbol{v})=\int_{\Omega} \boldsymbol{u} \cdot \boldsymbol{v} d V .
$$

In this work, all fields (and the corresponding spaces) are real since we have no absorption or complex boundary conditions.

The solution $\boldsymbol{E}$ of Maxwell's equations actually satisfies $\nabla \cdot \boldsymbol{E}=0$ in $\Omega$ so we define

$$
X=\left\{\boldsymbol{u} \in H_{0}(\operatorname{curl} ; \Omega) \mid \nabla \cdot \boldsymbol{u}=0 \text { in } \Omega\right\} .
$$

Using this space, the weak form of the eigenvalue problem (1.1)-(1.3) is to find $\boldsymbol{E} \in X, \boldsymbol{E} \neq 0$ and $\lambda \in \mathbb{R}$ such that

$$
(\nabla \times \boldsymbol{E}, \nabla \times \boldsymbol{\phi})=\lambda(\boldsymbol{E}, \boldsymbol{\phi}) \quad \forall \boldsymbol{\phi} \in X .
$$

The weak formulation of the source problem (1.4)-(1.6) is to find $\boldsymbol{E} \in X$ such that

$$
(\nabla \times \boldsymbol{E}, \nabla \times \phi)-k^{2}(\boldsymbol{E}, \boldsymbol{\phi})=-(\boldsymbol{J}, \boldsymbol{\phi}) \quad \forall \boldsymbol{\phi} \in X .
$$

For a general $\boldsymbol{J}$ with $\nabla \cdot \boldsymbol{J} \neq 0$, we cannot have $\nabla \cdot \boldsymbol{E}=0$. In this case we can use the Helmholtz decomposition to split the more general source problem into a problem like (1.8) and another, simpler, Poisson problem (see [22]).

We can rewrite both of these problems as an operator equation. We define the operator

$$
A:\left(L^{2}(\Omega)\right)^{3} \rightarrow\left(L^{2}(\Omega)\right)^{3}
$$

as follows. For given $\boldsymbol{u} \in\left(L^{2}(\Omega)\right)^{3}, A \boldsymbol{u} \in X$ satisfies

$$
(\nabla \times A \boldsymbol{u}, \nabla \times \boldsymbol{\phi})=(\boldsymbol{u}, \boldsymbol{\phi}) \quad \forall \boldsymbol{\phi} \in X .
$$

To establish that $A$ is well defined, we note that by the Friedrichs inequality [19] the bilinear form $a(\boldsymbol{u}, \boldsymbol{v})=(\nabla \times \boldsymbol{u}, \nabla \times \boldsymbol{v})$ is coercive on $X$ and hence by the Lax-Milgram Lemma $A \boldsymbol{u}$ is well defined (in fact we could even take $\boldsymbol{u} \in X^{\prime}$ where $X^{\prime}$ is the $\left(L^{2}(\Omega)\right)^{3}$ dual space of $\left.X\right)$.

Using the operator $A$ we can rewrite the eigenvalue problem (1.7) as the problem of finding $\boldsymbol{E} \in X, \boldsymbol{E} \neq 0$ and $\lambda \in \mathbb{R}$ such that

$$
\boldsymbol{E}=\lambda A \boldsymbol{E} .
$$

Letting $\mu=\frac{1}{\lambda}$ (possible since $\lambda \neq 0$ ) we obtain

$$
A \boldsymbol{E}=\mu \boldsymbol{E} .
$$

Now we note that $A$ is self-adjoint and compact. The latter property follows since $A$ is continuous from $\left(L^{2}(\Omega)\right)^{3}$ into $X$ and the Weber compactness result (see, for example, [20]) shows that $X$ is compactly embedded in $\left(L^{2}(\Omega)\right)^{3}$. Hence, as we have already noted, the existence of eigenvalues $\mu_{n}, n=1, \ldots$ of (1.10) can be verified.

Written as an operator equation, the source problem (1.8) becomes the problem of finding $\boldsymbol{E} \in X$ such that

$$
\boldsymbol{E}-k^{2} A \boldsymbol{E}=-A \boldsymbol{J}
$$


Using the Fredholm alternative, existence for this problem can also be verified using the compactness of $A$.

Now suppose that we discretize $H_{0}(\operatorname{curl} ; \Omega)$ using edge finite elements (details of this construction will be given in Section (3). This results in a family of finite element spaces

$$
V_{h} \subset H_{0}(\operatorname{curl} ; \Omega)
$$

parametrized by the mesh size $h>0$. A critical property of the edge spaces is that there is also a scalar space

$$
S_{h} \subset H_{0}^{1}(\Omega)
$$

such that $\nabla S_{h} \subset V_{h}$.

We cannot easily approximate $X$ by an interior approximation because of the difficulty of satisfying the divergence constraint. Considerations of mixed methods 23, 10, 22] or stabilized formulations 27] for (1.7) or (1.8) leads to defining

$$
X_{h}=\left\{\boldsymbol{u}_{h} \in V_{h} \mid\left(\boldsymbol{u}_{h}, \nabla \xi_{h}\right)=0 \forall \xi_{h} \in S_{h}\right\} .
$$

Note that $X_{h} \not \subset X$. The above definition allows us to write the following orthogonal decomposition:

$$
V_{h}=X_{h} \oplus \nabla S_{h} .
$$

Using $X_{h}$, the discrete eigenvalue problem corresponding to (1.7) is to find $\boldsymbol{E}_{h} \in$ $X_{h}, \boldsymbol{E}_{h} \neq 0$ and $\lambda_{h} \in \mathbb{R}$ such that

$$
\left(\nabla \times \boldsymbol{E}_{h}, \nabla \times \boldsymbol{\phi}_{h}\right)=\lambda_{h}\left(\boldsymbol{E}_{h}, \boldsymbol{\phi}_{h}\right) \quad \forall \phi_{h} \in X_{h} .
$$

The discrete source problem corresponding to (1.8) is to find $\boldsymbol{E}_{h} \in X_{h}$ such that

$$
\left(\nabla \times \boldsymbol{E}_{h}, \nabla \times \boldsymbol{\phi}_{h}\right)-k^{2}\left(\boldsymbol{E}_{h}, \boldsymbol{\phi}_{h}\right)=-\left(\boldsymbol{J}, \boldsymbol{\phi}_{h}\right) \quad \forall \boldsymbol{\phi}_{h} \in X_{h} .
$$

Note that practical calculations are usually done using $V_{h}$ in place of $X_{h}$. Thus for the eigenvalue problem we would actually compute $\boldsymbol{E}_{h} \in V_{h}, \boldsymbol{E}_{h} \neq 0$ and $\lambda_{h} \in \mathbb{R}$ such that

$$
\left(\nabla \times \boldsymbol{E}_{h}, \nabla \times \boldsymbol{\phi}_{h}\right)=\lambda_{h}\left(\boldsymbol{E}_{h}, \phi_{h}\right) \quad \forall \phi_{h} \in V_{h} .
$$

However, using the decomposition (1.12) we can choose $\phi=\nabla \xi_{h}$ for $\xi_{h} \in S_{h}$ and conclude that

$$
\lambda_{h}\left(\boldsymbol{E}_{h}, \nabla \xi_{h}\right)=0, \quad \forall \xi_{h} \in S_{h} .
$$

Since physical eigenvalues are nonzero, we can conclude that for the interesting eigenvalues $\boldsymbol{E}_{h} \in X_{h}$ and it suffices to analyze (1.13). In the same way, instead of (1.14) we would usually compute $\boldsymbol{E}_{h} \in V_{h}$ such that

$$
\left(\nabla \times \boldsymbol{E}_{h}, \nabla \times \boldsymbol{\phi}_{h}\right)-k^{2}\left(\boldsymbol{E}_{h}, \boldsymbol{\phi}_{h}\right)=-\left(\boldsymbol{J}, \boldsymbol{\phi}_{h}\right) \quad \forall \boldsymbol{\phi}_{h} \in V_{h} .
$$

Again choosing $\phi_{h}=\nabla \xi_{h}$ for $\xi_{h} \in S_{h}$ and using the fact that $\boldsymbol{J}$ is assumed to be divergence free, we conclude that $\boldsymbol{E}_{h} \in X_{h}$ and it suffices to analyze (1.14).

Now we can rewrite (1.13) and (1.14) as discrete operator equations. We define

$$
A_{h}:\left(L^{2}(\Omega)\right)^{3} \rightarrow\left(L^{2}(\Omega)\right)^{3}
$$

as follows. Given $\boldsymbol{u} \in\left(L^{2}(\Omega)\right)^{3}$, we define $A_{h} \boldsymbol{u} \in X_{h}$ to satisfy

$$
\left(\nabla \times A_{h} \boldsymbol{u}, \nabla \times \phi_{h}\right)=\left(\boldsymbol{u}, \phi_{h}\right) \quad \forall \phi_{h} \in X_{h} .
$$


We shall prove (in Section 4) that $A_{h}$ is well defined. Assuming this for the moment we can rewrite the eigenvalue problem (1.13) as the problem of finding $\boldsymbol{E}_{h} \in X_{h}$, $\boldsymbol{E}_{h} \neq 0$ and $\mu_{h} \in \mathbb{R}$ such that

$$
A_{h} \boldsymbol{E}_{h}=\mu_{h} \boldsymbol{E}_{h} .
$$

As before, $\mu_{h}=\frac{1}{\lambda_{h}}$.

The discrete source problem (1.14) is equivalent to finding $\boldsymbol{E}_{h} \in X_{h}$ such that

$$
\boldsymbol{E}_{h}-k^{2} A_{h} \boldsymbol{E}_{h}=-A_{h} \boldsymbol{J} .
$$

To prove convergence for either problem, we apply the theory of pointwise convergent collectively compact operators (see Section 2 for details). Let $\left\{h_{n}\right\}_{n=0}^{\infty}$ denote a refinement path so that

$$
h_{0}>h_{1}>h_{2}>\cdots>0
$$

and $h_{n} \rightarrow 0$ as $n \rightarrow \infty$. The numbers $h_{n}$ index a sequence of progressively finer meshes used to approximate the problem. Let $\Lambda=\left\{h_{n}, n=0,1,2, \ldots\right\}$. For future use, we define the set

$$
W=\cup_{h \in \Lambda} X_{h} \subset H_{0}(\operatorname{curl} ; \Omega) .
$$

This set plays the role of $X$ for the discrete problem. We also can define the collection of operators

$$
\mathcal{A}=\left\{A_{h}:\left(L^{2}(\Omega)\right)^{3} \rightarrow\left(L^{2}(\Omega)\right)^{3}, h \in \Lambda\right\} .
$$

To apply the abstract convergence theory, we need to verify:

(1) $\mathcal{A}$ is collectively compact.

(2) $\mathcal{A}$ is pointwise convergent by which we mean that for each $\boldsymbol{f} \in\left(L^{2}(\Omega)\right)^{3}$, we have

$$
A_{h_{n}} f \rightarrow A f
$$

strongly in $\left(L^{2}(\Omega)\right)^{3}$ as $n \rightarrow \infty$.

In Section 4 we verify both these properties for the two edge finite element spaces outlined in Section 3. We note that Levillain [21] has already verified condition (2) but, for completeness, we shall give a different proof here.

Since we are dealing with a collectively compact set of pointwise convergent operators, we can then verify existence and uniqueness, as well as convergence for each of the two problems discussed here.

An outline of the paper is as follows. In Section 2 we recall some facts about collectively compact operators. In Section 3 we outline the two families of finite elements under consideration. In Section 4 we verify that $\mathcal{A}$ satisfies properties (1.1) and (1.2). Finally in Section 5 we give error estimates for each problem, and make some conclusions in Section 6 .

\section{Collectively compact operators}

For a general discussion of collectively compact operators see Anselone [2]. Here we present some well-known results for operators of this type. First we recall the following definition. 
Definition 2.1 (Collectively Compact Operators). A set $\mathcal{A}=\left\{A_{h}: \mathcal{X} \rightarrow \mathcal{Y}\right.$, $h \in \Lambda$ \} of linear operators mapping a normed space $\mathcal{X}$ into a normed space $\mathcal{Y}$ is called collectively compact if for each bounded set $U \subset \mathcal{X}$ the image set

$$
\mathcal{A}(U)=\left\{A_{h} u: u \in U, A_{h} \in \mathcal{A}\right\}
$$

is relatively compact.

Now let us suppose $\mathcal{A}$ is a collection of collectively compact, self-adjoint and pointwise convergent operators $A_{h}$. Suppose the operators converge pointwise to an operator $A$ which is compact and self-adjoint.

First we summarize some known results for the eigenvalue problem. Let us suppose that $\mu$ is an eigenvalue of $A$ of multiplicity $m$. Let $E(\mu)$ denote the eigenspace corresponding to the eigenvalue $\mu$. Osborn [26] proves the following theorem (we have collected results in the paper and assume that the operators are self-adjoint):

Theorem 2.2. Suppose $\epsilon>0$ is such that the disk of radius $\epsilon$ about $\mu$ contains no other eigenvalues of $A$. Then for $h$ small enough the disk of radius $\epsilon$ centered at $\mu$ contains precisely $m$ eigenvalues of the discrete problem denoted $\mu_{h, j}, j=1, \ldots, m$. The dimension of $E(\mu)$ is equal to that of $\bigoplus_{j=1}^{m} E\left(\mu_{h, j}\right)$. Finally, for $1 \leq j \leq m$ there is a constant $C$ such that

$$
\left|\mu-\mu_{h, j}\right| \leq C\left\{\sum_{\ell, j=1}^{m}\left|\left(\left(A-A_{h}\right) \phi_{j}, \phi_{\ell}\right)\right|+\left\|\left.\left(A-A_{h}\right)\right|_{E(\mu)}\right\|^{2}\right\} .
$$

Here $\left\{\phi_{j}\right\}_{j=1}^{m}$ is an $\left(L^{2}(\Omega)\right)^{3}$ orthonormal basis for $E(\mu)$ and $\left.\left(A-A_{h}\right)\right|_{E(\mu)}$ is the restriction of $\left(A-A_{h}\right)$ to $E(\mu)$. We recall that $\|\cdot\|$ denotes the $\left(L^{2}(\Omega)\right)^{3}$ norm.

Remark 1. Osborn also provides an estimate for the distance of $E(\mu)$ to $\bigoplus_{j=1}^{m} E\left(\mu_{h, j}\right)$.

Next we discuss the source problem. The result we give is an obvious extension of Theorems 10.8 and 10.9 of [18 to allow for the fact that the right-hand side of (1.17) approximates the right-hand side of (1.11). Similar results are in [2].

Theorem 2.3. For $h$ sufficiently small (or for $h_{0}$ sufficiently small in the definition of $\Lambda)$ we have that $\left(I-k^{2} A_{h}\right)$ is invertible and

$$
\left\|\left(I-k^{2} A_{h}\right)^{-1}\right\| \leq \frac{1+k^{2}\left\|\left(I-k^{2} A\right)^{-1} A_{h}\right\|}{1-k^{4}\left\|\left(I-k^{2} A\right)^{-1}\left(A_{h}-A\right) A_{h}\right\|} .
$$

Furthermore if $\boldsymbol{E}$ satisfies (1.11) and $\boldsymbol{E}_{h}$ satisfies (1.17) we have

$$
\left\|\boldsymbol{E}-\boldsymbol{E}_{h}\right\| \leq\left\|\left(I-k^{2} A_{h}\right)^{-1}\right\| E_{1}+\left\|\left(I-k^{2} A\right)^{-1}\right\| E_{2},
$$

where

$$
E_{1}=\frac{k^{2}\left\|\left(A_{h}-A\right) \boldsymbol{J}\right\|+k^{4}\left\|\left(A_{h}-A\right) A \boldsymbol{E}\right\|}{1-k^{4}\left\|\left(I-k^{2} A\right)^{-1}\left(A_{h}-A\right) A\right\|}
$$

and

$$
E_{2}=\left\|\left(A_{h}-A\right) \boldsymbol{J}\right\| .
$$

Remark 2. A sufficient condition for invertibility of $\left(I-k^{2} A_{h}\right)$ is that

$$
k^{4}\left\|\left(I-k^{2} A\right)^{-1}\left(A_{h}-A\right) A_{h}\right\|<1 .
$$


Corollary 10.7 of [18] shows that once we have verified properties (1) and (2) from the introduction we know that

$$
\left(A_{h}-A\right) A_{h} \rightarrow 0 \quad \text { strongly as } h \rightarrow 0,
$$

hence condition (2.6) is satisfied for all $h$ sufficiently small. Note that we have explicitly given the $k$ dependence of the estimates. A hope (not realized in this study) is that the $k$ dependence of the error could be derived by this theory. See Ihlenburg 13 for a discussion of $k$ dependence of error estimates for the related one-dimensional Helmholtz equation.

\section{Details of the finite element method}

In this paper we shall consider two standard edge element spaces due to Nédélec 23]. Extensions to more general $h$-version elements, including those introduced by Demkowicz and Vardapetyan in [9, 29], or the second family of edge elements on tetrahedra 25] can be proved in the same way.

We start by covering $\Omega$ by a regular mesh consisting either of tetrahedra or cubes (but not both in one mesh). In the latter case we obviously restrict considerably the domains that can be covered. Let us denote the mesh by $\tau_{h}$ where $h$ is the maximum diameter of the elements in $\tau_{h}$. In addition, for our proof of the discrete compactness property, we need a weak quasi-uniformity restriction. Let $h_{K}$ denote the diameter of the smallest sphere containing the element $K \in \tau_{h}$ and let

$$
h_{\min }=\min _{K \in \tau_{h}} h_{K} .
$$

We say the mesh is weakly quasi-uniform if there is a constant $\mu^{*}$ with $0<\mu^{*}<1$ such that

$$
h h_{\min }^{-\mu^{*}} \rightarrow 0 \text { as } h \rightarrow 0 .
$$

Note that if the mesh is weakly quasi-uniform for some $\mu^{*}>0$, then it is also weakly quasi-uniform for any $\mu$ with $0<\mu \leq \mu^{*}$, since

$$
h h_{\min }^{-\mu}=\left(h h_{\min }^{-\mu^{*}}\right) h_{\min }^{\mu^{*}-\mu} \rightarrow 0 \quad \text { as } h \rightarrow 0 .
$$

Hence a quasi-uniform mesh is weakly quasi-uniform. The assumption of weak quasi-uniformity allows some nonuniform mesh refinement (but, unfortunately, not a geometric refinement).

3.1. Tetrahedral elements. Here we assume that the mesh is a regular and weakly quasi-uniform collection of tetrahedra. In order to define the original curl conforming space of Nédélec [23, we let $P_{k}$ denote the standard space of polynomials of total degree less than or equal to $k$, and let $\tilde{P}_{k}$ denote the space of homogeneous polynomials of order $k$. Now we define $S_{k} \subset\left(P_{k}\right)^{3}$ and $R_{k} \subset\left(P_{k}\right)^{3}$ by

$$
\begin{aligned}
S_{k} & =\left\{\boldsymbol{p} \in\left(\tilde{P}_{k}\right)^{3} \mid \boldsymbol{p}(\boldsymbol{x}) \cdot \boldsymbol{x}=0\right\}, \\
R_{k} & =\left(P_{k-1}\right)^{3} \oplus S_{k} .
\end{aligned}
$$

For example, if $k=1$, then a polynomial $p \in R_{k}$ has the form

$$
\boldsymbol{p}(\boldsymbol{x})=\boldsymbol{\alpha}+\boldsymbol{\beta} \times \boldsymbol{x},
$$

where $\boldsymbol{\alpha}$ and $\boldsymbol{\beta}$ are constant vectors [24]. Following [23], for given $p \geq 1$, we define

$$
U_{h}=\left\{\boldsymbol{v}_{h} \in H(\operatorname{curl} ; \Omega)\left|\boldsymbol{v}_{h}\right|_{K} \in R_{p} \quad \forall K \in \tau_{h}\right\} .
$$


To define the degrees of freedom in $U_{h}$ we define the following moments. If $K \in \tau_{h}$ with general edge $e$ and face $f$ and if $\boldsymbol{\tau}$ is a unit vector parallel to $e$, we define

$$
\begin{aligned}
M_{e}(\boldsymbol{u}) & =\left\{\int_{e} \boldsymbol{u} \cdot \boldsymbol{\tau} q d s \quad \forall q \in P_{p-1}(e) \text { for the six edges } e \text { of } K\right\}, \\
M_{f}(\boldsymbol{u}) & =\left\{\int_{f} \boldsymbol{u} \times \boldsymbol{\nu} \cdot \boldsymbol{g} d A \quad \forall \boldsymbol{g} \in\left(P_{p-2}(f)\right)^{2} \text { for all four faces } f \text { of } K\right\}, \\
M_{K}(\boldsymbol{u}) & =\left\{\int_{K} \boldsymbol{u} \cdot \boldsymbol{g} d \boldsymbol{x} \quad \forall \boldsymbol{g} \in\left(P_{p-3}(K)\right)^{2}\right\} .
\end{aligned}
$$

These moments are defined if $\boldsymbol{u} \in H^{r}(\Omega)$ and $\nabla \times \boldsymbol{u} \in H^{r}(\Omega)$ for some $r>1 / 2$, or if $u \in H^{1+\epsilon}(\Omega)$ for some $\epsilon>0$ (see [1]). Unfortunately the moments are not defined in general for $\boldsymbol{u} \in H^{1}(\Omega)$, which is a complicating factor in the analysis.

Nédélec [23] shows that the above three sets of degrees of freedom are unisolvent and curl conforming. Using these degrees of freedom we can define an interpolant denoted $r_{h} \boldsymbol{u} \in U_{h}$ for any function $\boldsymbol{u}$ for which (3.1)-(3.3) are defined. On each $K \in \tau_{h}$ we pick $\left.r_{h} \boldsymbol{u}\right|_{K} \in R_{p}$ such that

$$
M_{e}\left(\boldsymbol{u}-r_{h} \boldsymbol{u}\right)=M_{f}\left(\boldsymbol{u}-r_{h} \boldsymbol{u}\right)=M_{K}\left(\boldsymbol{u}-r_{h} \boldsymbol{u}\right)=\{0\} .
$$

To approximate functions in $H_{0}(\operatorname{curl} ; \Omega)$ we define

$$
V_{h}=\left\{\boldsymbol{u}^{h} \in U_{h} \mid \boldsymbol{\nu} \times \boldsymbol{u}^{h}=0 \quad \text { on } \Gamma\right\} .
$$

The constraint $\boldsymbol{\nu} \times \boldsymbol{u}^{h}=0$ on $\Gamma$ is easily implemented by taking the degrees of freedom associated with edges or faces on $\Gamma$ to be zero [12].

The associated scalar space is

$$
S_{h}=\left\{q_{h} \in H_{0}^{1}(\Omega)\left|q_{h}\right|_{K} \in P_{p}\right\} .
$$

With this choice we have $\nabla S_{h} \subset V_{h}$. As we shall see, this space is also the correct space to define discrete divergence-free vector fields.

3.2. Quadrilateral elements. In this subsection, let the mesh $\tau_{h}$ be a regular and weakly quasi-uniform covering of $\Omega$ using hexahedra with each edge parallel to one of the coordinate axes. Let $Q_{p, l, m}$ be the space of polynomials of maximum degree $p$ in $x, l$ in $y$ and $m$ in $z$. Corresponding to the simplicial elements discussed in the previous subsection, Nédélec has proposed some elements on hexahedra [23]. In this case, for given $p \geq 1$, we define

$$
U_{h}=\left\{\boldsymbol{u}^{h} \in H_{0}(\operatorname{curl} ; \Omega)\left|\boldsymbol{u}^{h}\right|_{K} \in Q_{p-1, p, p} \times Q_{p, p-1, p} \times Q_{p, p, p-1} \quad \forall K \in \tau_{h}\right\}
$$

with the following degrees of freedom. If $K$ is a cube with general edge $e$ and face $f$, and if $\boldsymbol{\tau}$ is a unit vector along $e$ we define

$$
\begin{aligned}
& M_{e}(\boldsymbol{u})=\left\{\int_{e} \boldsymbol{u} \cdot \boldsymbol{\tau} g d s \mid g \in P_{p-1} \quad \forall e \in \partial K\right\} \\
& M_{f}(\boldsymbol{u})=\left\{\int_{f} \boldsymbol{\nu} \times \boldsymbol{u} \cdot \boldsymbol{g} d A \mid \boldsymbol{g}=\left(g_{1}, g_{2}\right)^{T}\right. \text { where } \\
& M_{K}(\boldsymbol{u})=\left\{\int_{K} \boldsymbol{u} \cdot \boldsymbol{g} d V \mid \boldsymbol{g}=\left(g_{1}, g_{2}, g_{3}\right) \in Q_{p-1, p-2, p-2}, g_{2} \in Q_{p-1, p-2} \quad \forall f \in \partial K\right\} \\
&\left.\times Q_{p-2, p-1, p-2} \times Q_{p-2, p-2, p-1}\right\} .
\end{aligned}
$$


Nédélec shows that (3.6)-(3.8) are curl conforming and unisolvent for elements in $U_{h}$ given by (3.5) [23]. Now we can take $V_{h} \subset H_{0}(\operatorname{curl} ; \Omega)$ to be

$$
V_{h}=\left\{\boldsymbol{v}_{h} \in U_{h} \mid \boldsymbol{\nu} \times \boldsymbol{v}_{h}=0 \text { on } \Gamma\right\} .
$$

The corresponding space of scalar functions is

$$
S_{h}=\left\{q_{h} \in H_{0}^{1}(\Omega)\left|q_{h}\right|_{K} \in Q_{k, k, k} \quad \forall K \in \tau_{h}\right\} .
$$

3.3. Interpolation error estimates. Using either the tetrahedral or hexahedral edge element families presented above we have the following result.

Theorem 3.1. (a) For any sufficiently smooth $p \in H_{0}^{1}(\Omega)$ we have that $r_{h} \nabla p=$ $\nabla P_{h} p$ where $P_{h}$ is a suitable interpolation operator for $S_{h}$. Furthermore for any $\delta>3 / 2$ there is a constant $C$ depending on $\delta$ but not $p$ such that

$$
\left\|\nabla\left(P_{h} p-p\right)\right\| \leq C h^{\delta-1}\|\nabla p\|_{\delta-1} .
$$

(b) There exists a constant $C=C(\epsilon)$ such that

$$
\left\|\boldsymbol{u}-r_{h} \boldsymbol{u}\right\| \leq C h\|\boldsymbol{u}\|_{1+\epsilon}
$$

(c)

for any $\epsilon>0$.

$$
\left\|\boldsymbol{u}-r_{h} \boldsymbol{u}\right\|+\left\|\nabla \times\left(\boldsymbol{u}-r_{h} \boldsymbol{u}\right)\right\| \leq C h^{p}\left(\|\boldsymbol{u}\|_{H^{p}(\Omega)}+\|\nabla \times \boldsymbol{u}\|_{H^{p}(\Omega)}\right) .
$$

Proof. The equality in part (a) is classical and proved for example in 12, 11]. The error estimate is then standard for the interpolation operator in $S_{h}$ (the restriction on $\delta$ ensures that $p$ is continuous and hence can be interpolated).

Part (c) is proved in [1], and part (b) is proved in the same way using a scaling argument.

We also recall the following result proved in $[5]$ for general piecewise polynomials.

Lemma 3.2. Suppose the mesh is regular, then for any $\boldsymbol{u}_{h} \in X_{h}$ and $0 \leq \sigma \leq 1 / 2$ there is a constant $C$ independent of $h$ and $\boldsymbol{u}_{h}$ such that

$$
\left\|\nabla \times \boldsymbol{u}_{h}\right\|_{H^{\sigma}(\Omega)} \leq C h_{\min }^{-\sigma}\left\|\nabla \times \boldsymbol{u}_{h}\right\| .
$$

\section{Discrete and collective compactness}

In this section we prove that the collection of operators $\mathcal{A}$ introduced in (1.19) is collectively compact and pointwise convergent. The main tool in this analysis is the discrete compactness property of the spaces $\left\{X_{h}\right\}_{h \in \Lambda}$.

To date discrete compactness has only been proved for the lowest order Nédélec space of edge elements in tetrahedra by Kikuchi [15, 16, 17] (see [14] for the corresponding $2 \mathrm{D}$ result). The first theorem here shows that, under the weak quasiuniformity assumption on the mesh, higher order edge element spaces, including elements on hexahedra, also satisfy the discrete compactness property.

The proof of this result is based on a regularity result due to Costabel and Dauge 7] which we now state.

For given $\boldsymbol{f} \in X^{\prime}$ with $\nabla \cdot \boldsymbol{f}=0$ in $\Omega$, let $\boldsymbol{u} \in X$ satisfy

$$
\nabla \times \nabla \times \boldsymbol{u}=\boldsymbol{f} \quad \text { in } \Omega .
$$

For a given domain $\Omega$ there is a constant $\sigma_{0}>0$ such that for all $\sigma$ with $0 \leq \sigma<\sigma_{0}$ and $\boldsymbol{f} \in\left(H^{\sigma-1}(\Omega)\right)^{3}$ we can write

$$
\boldsymbol{u}=\boldsymbol{u}^{*}+\nabla \chi
$$


where $\boldsymbol{u}^{*} \in\left(H^{\sigma+1}(\Omega)\right)^{3}$ and $\chi \in H_{0}^{1}(\Omega)$ with $\Delta \chi \in H^{\sigma}(\Omega)$. In addition

$$
\begin{array}{r}
\left\|\boldsymbol{u}^{*}\right\|_{H^{1+\sigma}(\Omega)}+\|\chi\|_{H^{1}(\Omega)} \leq C\|\boldsymbol{f}\|_{H^{\sigma-1}(\Omega)}, \\
\|\Delta \chi\|_{L^{2}(\Omega)} \leq C\|\boldsymbol{f}\|_{H^{-1}(\Omega)} .
\end{array}
$$

The decomposition (4.2) is not orthogonal. Precise estimates for $\sigma_{0}$ involving edge and corner exponents of the domain are given in Theorem 8.6 of [7. We note also that the same theorem gives an expansion for $\chi$ in terms of edge and corner singularities.

Note that since $\Delta \chi \in L^{2}(\Omega)$ and $\chi \in H_{0}^{1}(\Omega)$ we can actually conclude that $\chi \in H^{3 / 2}(\Omega)$ (see, for example, the proof of Theorem 2 in [6]). This is not sufficient for our purposes, and so we assume that $\Omega$ is such that there is an $\epsilon>0$ such that $\chi \in H^{3 / 2+\epsilon}(\Omega)$, and

$$
\|\chi\|_{H^{3 / 2+\epsilon}(\Omega)} \leq C\|\Delta \chi\| .
$$

Of course this holds for a convex domain (with $\epsilon=1 / 2$ ) and so this assumption constitutes a restriction on the interior angles of $\Omega$.

Now we are ready to state and prove the discrete compactness property.

Theorem 4.1. Assume that the regularity results given in (4.3) and (4.4) hold. Suppose in addition that the mesh is regular and weakly quasi-uniform. Finally, suppose that the sequence $\left\{\boldsymbol{u}_{n}\right\}_{n=1}^{\infty}$ has the following properties:

- $\left\{\boldsymbol{u}_{n}\right\}_{n=1}^{\infty}$ is a bounded sequence in $H_{0}(\operatorname{curl} ; \Omega)$;

- $\boldsymbol{u}_{n} \in X_{h_{n}}$ for each $n$ and $h_{n} \rightarrow 0$ as $n \rightarrow 0$.

Then there is a subsequence, still denoted $\left\{\boldsymbol{u}_{n}\right\}_{n=1}^{\infty}$, and a function $\boldsymbol{u} \in X$ such that

$$
\boldsymbol{u}_{n} \rightarrow \boldsymbol{u}
$$

strongly in $\left(L^{2}(\Omega)\right)^{3}$ and weakly in $X$.

Remark 3. When this theorem holds we say $\left\{X_{h}\right\}_{h \in \Lambda}$ has the discrete compactness property. As we have already pointed out in the Introduction, this is already known for edge elements on a tetrahedral mesh when $p=1$ [17.

Proof. Using the Helmholtz decomposition we may write

$$
\boldsymbol{u}_{n}=\boldsymbol{u}^{n}+\nabla p^{n},
$$

where $p^{n} \in H_{0}^{1}(\Omega)$ satisfies $\left(\nabla p^{n}, \nabla \xi\right)=\left(\boldsymbol{u}_{n}, \nabla \xi\right)$ for all $\xi \in H_{0}^{1}(\Omega)$, and $\boldsymbol{u}^{n} \in X$ satisfies

$$
\nabla \times \boldsymbol{u}^{n}=\nabla \times \boldsymbol{u}_{n} \text { in } \Omega
$$

or

$$
\nabla \times \nabla \times \boldsymbol{u}^{n}=\nabla \times\left(\nabla \times \boldsymbol{u}_{h}\right) \text { in } \Omega,
$$

where this equation is understood in the sense of distributions. But $\nabla \times \boldsymbol{u}_{n} \in$ $\left(H^{\epsilon}(\Omega)\right)^{3}$ for any $0 \leq \epsilon<1 / 2$ since $\nabla \times \boldsymbol{u}_{n}$ is a piecewise polynomial vector. Hence by the regularity assumption

$$
\boldsymbol{u}^{n}=\boldsymbol{\omega}^{n}+\nabla \chi^{n}
$$

for $\boldsymbol{\omega}^{n} \in\left(H^{1+\epsilon}(\Omega)\right)^{3}$ provided $0<\epsilon<\sigma_{0}$. But this implies that $r_{h_{n}} \boldsymbol{\omega}^{* n}$ is welldefined.

By the regularity assumption on $\chi$ given at the beginning of the section, $\chi \in$ $H^{3 / 2+\epsilon}(\Omega)$ for some $\epsilon>0$ and hence $r_{h_{n}} \nabla \chi$ is well defined and by Theorem [3.1] (a) $r_{h_{n}} \nabla \chi^{n}=\nabla P_{h_{n}} \chi^{n}$. An alternative argument to this is that since $\chi$ consists of 
edge and corner singularities for Laplace's equation we can check that if $\chi=r^{\alpha}$, where $r=|\boldsymbol{x}|$, then $r_{h} \nabla \chi$ is well defined for $\alpha>0$ since line integrals of $\partial \chi / \partial r$ are well defined in the case.

Using (4.5) and the fact that $r_{h_{n}} \boldsymbol{u}_{n}=\boldsymbol{u}_{n}$, we have that

$$
\boldsymbol{u}_{n}=r_{h_{n}} \boldsymbol{u}^{n}+\nabla P_{h_{n}} p^{n} \text {. }
$$

But $\boldsymbol{u}_{n} \in X_{h_{n}}$ and $\boldsymbol{u}^{n} \in X$ so that

$$
\left(\nabla P_{h_{n}} p^{n}, \nabla P_{h_{n}} p^{n}\right)=\left(\boldsymbol{u}_{n}-r_{h_{n}} \boldsymbol{u}^{n}, \nabla P_{h_{n}} p^{n}\right)=\left(\boldsymbol{u}^{n}-r_{h_{n}} \boldsymbol{u}^{n}, \nabla P_{h_{n}} p^{n}\right) .
$$

Thus $\left\|\nabla P_{h_{n}} p^{n}\right\| \leq\left\|\boldsymbol{u}^{n}-r_{h_{n}} \boldsymbol{u}^{n}\right\|$. Furthermore

$$
\boldsymbol{u}_{h}=\left(r_{h_{n}} \boldsymbol{u}^{n}-\boldsymbol{u}^{n}\right)+\boldsymbol{u}^{n}+\nabla P_{h_{n}} p^{n} .
$$

The sequence $\left\{\boldsymbol{u}^{n}\right\}_{n=1}^{\infty} \subset X$ and satisfies (using the continuous Friedrichs inequality)

$$
\left\|\boldsymbol{u}^{n}\right\|+\left\|\nabla \times \boldsymbol{u}^{n}\right\| \leq C,
$$

and so by Weber's compactness result (see 20]) there is a sequence, still denoted $\left\{\boldsymbol{u}^{n}\right\}_{n=1}^{\infty}$ such that $\boldsymbol{u}^{n} \rightarrow \boldsymbol{u}$ strongly in $\left(L^{2}(\Omega)\right)^{3}$ and weakly in $X$ for some function $\boldsymbol{u} \in X$.

The discrete compactness property is thus proved if we can show that $\| \boldsymbol{u}^{n}-$ $r_{h_{n}} \boldsymbol{u}^{n} \| \rightarrow 0$ as $n \rightarrow \infty$. Using the definition of $\boldsymbol{\omega}^{n}$ and $\chi^{n}$

$$
r_{h_{n}} \boldsymbol{u}^{n}-\boldsymbol{u}^{n}=\left(r_{h_{n}} \boldsymbol{\omega}^{n}-\boldsymbol{\omega}^{n}\right)+\nabla\left(P_{h_{n}} \chi^{n}-\chi^{n}\right) .
$$

But by the assumed regularity of $\chi^{n}$,

$$
\left\|\nabla\left(P_{h_{n}} \chi^{n}-\chi^{n}\right)\right\| \leq C h_{n}^{1 / 2+\epsilon}\left\|\nabla \chi^{n}\right\|_{H^{1 / 2+\epsilon}(\Omega)} \leq C h_{n}^{1 / 2+\epsilon}\left\|\Delta \chi^{n}\right\| .
$$

However $\left\|\Delta \chi^{n}\right\| \leq C$ for each $n$, hence

$$
\left\|P_{h_{n}} \chi^{n}-\chi^{n}\right\|_{H^{1}(\Omega)} \rightarrow 0 \text { as } n \rightarrow \infty \text {. }
$$

Next we analyze $\left\|r_{h_{n}} \boldsymbol{\omega}^{n}-\boldsymbol{\omega}^{n}\right\|$. Using the error estimate of Theorem[3.1(c) and the regularity of $\boldsymbol{\omega}^{n}$ we have

$$
\left\|r_{h_{n}} \boldsymbol{\omega}^{n}-\boldsymbol{\omega}^{n}\right\| \leq C h_{n}\left\|\boldsymbol{\omega}^{n}\right\|_{H^{1+\epsilon}(\Omega)},
$$

and using the norm estimate (4.3) together with the assumed weak quasi-uniformity assumption (and hence the inverse estimate in Lemma 3.2 choosing $\varepsilon<1 / 2$ ) we have

$$
\left\|r_{h_{n}} \boldsymbol{\omega}^{n}-\boldsymbol{\omega}^{n}\right\| \leq C h_{n}\left\|\nabla \times \boldsymbol{u}_{n}\right\|_{H^{\epsilon}(\Omega)} \leq C h_{n} h_{\min , n}^{-\epsilon}\left\|\nabla \times \boldsymbol{u}_{n}\right\| \rightarrow 0
$$

as $n \rightarrow \infty$. Using (4.7) and (4.8) in (4.6) shows that $\left\|\boldsymbol{u}^{n}-r_{h_{n}} \boldsymbol{u}^{n}\right\| \rightarrow 0$ as $n \rightarrow \infty$ and completes the proof.

Next we show that the discrete compactness property implies a discrete Friedrichs inequality.

Corollary 4.2. Suppose $\left\{X_{h}\right\}_{h \in \Lambda}$ has the discrete compactness property. Then provided $\partial \Omega$ is simply connected there are constants $C>0$ independent of $h$ and $h_{0}$ such that for all $h \leq h_{0}$ and any $\boldsymbol{u}_{h} \in X_{h}$

$$
\left\|\boldsymbol{u}_{h}\right\| \leq C\left\|\nabla \times \boldsymbol{u}_{h}\right\|
$$

Remark 4. Assuming the convexity of $\Omega$, this result was proved in [23]. 
Proof. The proof is by contradiction. Suppose the result is false so that there is a sequence $\left\{\boldsymbol{u}_{h_{n}}\right\}_{n=1}^{\infty}$ such that $\boldsymbol{u}_{h_{n}} \in X_{h_{n}}$ and

$$
\left\|\boldsymbol{u}_{h_{n}}\right\|=1, \quad\left\|\nabla \times \boldsymbol{u}_{h_{n}}\right\|=\frac{1}{n}
$$

for $1 \leq n<\infty$. By the discrete compactness property there is a subsequence, still denoted $\left\{\boldsymbol{u}_{h_{n}}\right\}_{n=1}^{\infty}$ such that $\boldsymbol{u}_{h_{n}} \rightarrow \boldsymbol{u}$ strongly in $\left(L^{2}(\Omega)\right)^{3}$ and weakly in $H(\operatorname{curl} ; \Omega)$ for some $\boldsymbol{u} \in X$. Hence $\|\boldsymbol{u}\|=1$. However for any $\phi \in H_{0}(\operatorname{curl} ; \Omega)$,

$$
(\nabla \times \boldsymbol{u}, \nabla \times \boldsymbol{\phi})=\lim _{n \rightarrow \infty}\left(\nabla \times \boldsymbol{u}_{h_{n}}, \nabla \times \phi\right) \leq \frac{1}{n}\|\nabla \times \phi\| \rightarrow 0, \text { as } n \rightarrow \infty .
$$

Hence $\nabla \times \boldsymbol{u}=0$ in $\Omega$ and $\boldsymbol{u} \in X$ so $\boldsymbol{u}=0$ by the standard Friedrichs inequality. This is a contradiction.

Our next result concerns the embedding of $W$ into $\left(L^{2}(\Omega)\right)^{3}$.

Lemma 4.3. Suppose that $\left\{X_{h}\right\}_{h \in \Lambda}$ has the discrete compactness property. Then

$$
W \hookrightarrow\left(L^{2}(\Omega)\right)^{3} .
$$

Remark 5. In the case of $2 \mathrm{D}$ edge elements on triangles, this result was proved in 14]. We follow this proof.

Proof. Let $\left\{\boldsymbol{\omega}_{n}\right\}_{n=1}^{\infty}$ be a sequence in $W$ bounded in $H(\operatorname{curl} ; \Omega)$. For each $n, \boldsymbol{\omega}_{n} \in$ $X_{h_{n}}$ and we may assume $h_{n} \rightarrow 0$ as $n \rightarrow \infty$. For if $h_{n} \geq \delta>0 \Omega$, the fact that $h_{n} \in \Lambda$ implies only finitely many $h_{n}$ are used so $\{\boldsymbol{\omega}\}_{n=1}^{\infty}$ is contained in a finite dimensional vector space and hence a convergent subsequence can be extracted.

If $h_{n} \rightarrow 0$ as $n \rightarrow \infty$, we have that $\left\{\boldsymbol{\omega}_{n}\right\}_{n=1}^{\infty}$ satisfies the conditions for the discrete compactness property. Hence we can extract a subsequence converging strongly in $\left(L^{2}(\Omega)\right)^{3}$ and compactness of the embedding is proved.

Next we establish the collective compactness of $\mathcal{A}$.

Theorem 4.4. Suppose $\left\{X_{h}\right\}_{h \in \Lambda}$ has the discrete compactness property. Then $\mathcal{A}$ is collectively compact.

Proof. Here we verify Definition 2.1 by using the compact embedding of $W$ in $\left(L^{2}(\Omega)\right)^{3}$. Let $U \subset\left(L^{2}(\Omega)\right)^{3}$ be a bounded set. Then, if $\boldsymbol{u} \in U$, we know that $A_{h} \boldsymbol{u} \in X_{h}$ satisfies

$$
\left(\nabla \times A_{h} \boldsymbol{u}, \nabla \times \boldsymbol{\chi}_{h}\right)=\left(\boldsymbol{u}, \boldsymbol{\chi}_{h}\right) \quad \forall \chi_{h} \in X_{h},
$$

and it follows $\left\|\nabla \times A_{h} \boldsymbol{u}\right\| \leq C\|\boldsymbol{u}\|$. But using the discrete Friedrichs inequality in Corollary 4.2 we have

$$
\left\|A_{h} \boldsymbol{u}\right\|+\left\|\nabla \times A_{h} \boldsymbol{u}\right\| \leq C\|\boldsymbol{u}\| .
$$

Hence $\left\{A_{h} \boldsymbol{u}\right\}_{h \in \Lambda} \subset W$ and is bounded in $H($ curl; $\Omega)$. By the compact embedding of $W$ into $\left(L^{2}(\Omega)\right)^{3}$ a subsequence of $\left\{A_{h_{n}} \boldsymbol{u}\right\}_{n=1}^{\infty}$ converges strongly in $\left(L^{2}(\Omega)\right)^{3}$ and hence $\mathcal{A}(U)$ is precompact as required.

Finally we verify the pointwise convergence of $A_{h}$ to $A$ as $h \rightarrow 0$. This is a consequence of the density of $V_{h}$ in $H(\operatorname{curl} ; \Omega)$ and $S_{h}$ in $H_{0}^{1}(\Omega)$.

Theorem 4.5. Suppose the discrete compactness property holds. Let $\boldsymbol{f} \in\left(L^{2}(\Omega)\right)^{3}$ then

$$
A_{h} \boldsymbol{f} \rightarrow \text { Af } \quad \text { strongly in } H(\text { curl; } \Omega) \text { as } h \rightarrow 0 .
$$


Remark 6 . We only need strong convergence in $\left(L^{2}(\Omega)\right)^{3}$. The result is proved by Levillain [21]. We offer a different proof based on the mixed formulation of (1.15) as used in 14 to analyze the $2 \mathrm{D}$ waveguide problem.

Proof. For any $\boldsymbol{f} \in\left(L^{2}(\Omega)\right)^{3}, A_{h} \boldsymbol{f} \in X_{h}$ can be characterized by a mixed variational problem. In particular, $A_{h} f \in X_{h}$ and $p_{h} \in S_{h}$ satisfy

$$
\begin{aligned}
\left(\nabla \times A_{h} \boldsymbol{f}, \nabla \times \boldsymbol{\phi}_{h}\right)+\left(\nabla p_{h}, \boldsymbol{\phi}_{h}\right) & =\left(\boldsymbol{f}, \boldsymbol{\phi}_{h}\right) \quad \forall \boldsymbol{\phi}_{h} \in V_{h}, \\
\left(A_{h} \boldsymbol{f}, \nabla \xi_{h}\right) & =0 \quad \forall \xi_{h} \in S_{h} .
\end{aligned}
$$

Similarly $A f \in X$ and $p \in H_{0}^{1}(\Omega)$ satisfy

$$
\begin{aligned}
(\nabla \times A \boldsymbol{f}, \nabla \times \boldsymbol{\phi})+(\nabla p, \boldsymbol{\phi}) & =(\boldsymbol{f}, \boldsymbol{\phi}) \quad \forall \boldsymbol{\phi} \in H_{0}(\mathrm{curl} ; \Omega) \\
(A \boldsymbol{f}, \nabla \xi) & =0 \quad \forall \xi \in H_{0}^{1}(\Omega) .
\end{aligned}
$$

As pointed out in [23] 10, 22] this is a standard mixed finite element problem. The Babuška-Brezzi theory of convergence is applicable since

- The inf-sup condition holds. This states that there is a constant $C$ independent of $h$ such that for any $p_{h} \in S_{h}$

$$
\sup _{\phi_{h} \in V_{h}} \frac{\left(\nabla p_{h}, \phi_{h}\right)}{\left\|\phi_{h}\right\|_{H(\operatorname{curl} ; \Omega)}} \geq C\left\|p_{h}\right\|_{H^{1}(\Omega)}
$$

holds. This is proved in [23] and follows by taking $\phi_{h}=\nabla p_{h}$.

- If $\boldsymbol{u}_{h} \in X_{h}$, the discrete Friedrichs inequality in Corollary 4.2 shows that there is a constant $C>0$ independent of $h$ such that

$$
\left\|\boldsymbol{u}_{h}\right\| \leq C\left\|\nabla \times \boldsymbol{u}_{h}\right\| \quad \forall \boldsymbol{u}_{h} \in X_{h} .
$$

The usual theory of mixed methods then implies that

$$
\left\|A \boldsymbol{f}-A_{h} \boldsymbol{f}\right\|_{H(\operatorname{curl} ; \Omega)} \leq C\left\{\inf _{\chi_{h} \in X_{h}}\left\|\nabla \times\left(A \boldsymbol{f}-\chi_{h}\right)\right\|+\inf _{q_{h} \in S_{h}}\left\|\nabla\left(p-q_{h}\right)\right\|\right\} .
$$

But since $X_{h}$ differs from $V_{h}$ only by the addition of gradient terms (see (1.12)),

$$
\inf _{h} \in X_{h}\left\|\nabla \times\left(A f-\chi_{h}\right)\right\|=\inf _{\chi_{h} \in V_{h}}\left\|\nabla \times\left(A f-\chi_{h}\right)\right\| .
$$

The density of $V_{h}$ in $H_{0}(\operatorname{curl} ; \Omega)$ then implies that

$$
\lim _{h \rightarrow 0} \inf _{h}\left\|V_{h}\right\| \nabla \times\left(A f-\chi_{h}\right) \|=0 .
$$

Similarly, the density of $S_{h}$ in $H_{0}^{1}(\Omega)$ shows that

$$
\lim _{h \rightarrow 0} \inf _{q_{h} \in S_{h}}\left\|\nabla\left(p-q_{h}\right)\right\|=0 .
$$

If the functions $p$ and $A \boldsymbol{f}$ are smoother, we also have error estimates as the next theorem shows.

Theorem 4.6. If the discrete compactness property holds and if $A \boldsymbol{f} \in\left(H^{p+1}(\Omega)\right)^{3}$ and $p \in\left(H^{p+1}(\Omega)\right)^{3}$ (where Af and $p$ satisfy (4.10) ) we have

$$
\left\|A \boldsymbol{f}-A_{h} \boldsymbol{f}\right\|_{H(\operatorname{curl} ; \Omega)} \leq C h^{p}\left(\|A \boldsymbol{f}\|_{H^{p+1}(\Omega)}+\|p\|_{H^{p+1}(\Omega)}\right) .
$$

Remark 7. If $\nabla \cdot \boldsymbol{f}=0$, then $p=0$ (to see this take $\phi=\nabla p$ in (4.10), and hence we are reduced to needing the smoothness of $A f$ alone. 
Proof. The proof is directly from (4.11) using Theorem 3.1 and standard estimates for $S_{h}$.

\section{Convergence Results}

In the previous section we verified that $\mathcal{A}$ is collectively compact and pointwise convergent. Thus we can use the estimates (2.1) and (2.3) of Section 2 We note that the general theory shows that

$$
\left.\|\left(I-k^{2} A\right)^{-1}\left(A_{h}-A\right) A_{h}\right) \| \rightarrow 0 \quad \text { as } h \rightarrow 0 .
$$

Hence (2.6) is satisfied. It would be useful to obtain an order estimate for this term but we have been unable to do this due to the fact that $A_{h} \phi$ is not necessarily divergence free.

Theorem 5.1. If $\left\{X_{h}\right\}_{h \in \Lambda}$ has the discrete compactness property, then the following results hold:

- For the eigenvalue problem, suppose $\mu$ is an eigenvalue of multiplicity $\mathrm{m}$. Then there are exactly $m$ discrete eigenvalues $\mu_{h, j}, j=1, \ldots, m$ such that

$$
\left|\mu-\mu_{h, j}\right| \rightarrow 0,1 \leq j \leq m, \quad \text { as } h \rightarrow 0 .
$$

- Suppose $k^{2}$ is not an interior electric eigenvalue. Then the discrete solution $\boldsymbol{E}_{h}$ of the source problem (see (1.8) and (1.14)) is well defined for sufficiently small $h$, and

$$
\left\|\boldsymbol{E}-\boldsymbol{E}_{h}\right\| \rightarrow 0 \quad \text { as } h \rightarrow 0
$$

Proof. First we consider the eigenvalue problem. Using (1.9) and (1.15) we may write

$$
\begin{aligned}
\left(\left(A-A_{h}\right) \phi_{\ell}, \phi_{j}\right) & =\left(\nabla \times\left(A-A_{h}\right) \phi_{\ell}, \nabla \times A \phi_{j}\right) \\
& =\left(\nabla \times\left(A-A_{h}\right) \phi_{\ell}, \nabla \times\left(A-A_{h}\right) \phi_{j}\right) .
\end{aligned}
$$

Hence (1.7) may be rewritten

$$
\left|\mu-\mu_{h_{j}}\right| \leq C\left\{\max _{\ell}\left\|\nabla \times\left(A-A_{h}\right) \phi_{\ell}\right\|^{2}+\left\|\left.\left(A-A_{h}\right)\right|_{E(\mu)}\right\|^{2}\right\} .
$$

Since $E(\mu)$ is finite dimensional, the pointwise convergence of $A_{h}$ to $A$ in $H(\operatorname{curl} ; \Omega)$ shown in Lemma 4.5 proves that both terms on the right-hand side above vanish as $h \rightarrow 0$.

For the source problem, provided $h$ is small enough, $\left(I+k^{2} A_{h}\right)^{-1}$ exists (so $\boldsymbol{E}_{h}$ exists) and since (using the discrete Friedrichs inequality)

$$
\left\|A_{h}\right\|=\sup _{\phi \in\left(L^{2}(\Omega)\right)^{3}} \frac{\left\|A_{h} \phi\right\|}{\|\phi\|} \leq C \sup _{\phi \in\left(L^{2}(\Omega)\right)^{3}} \frac{\left\|\nabla \times A_{h} \phi\right\|}{\|\phi\|} \leq C,
$$

we know that for $h$ small enough

$$
\left\|\left(I-k^{2} A_{h}\right)^{-1}\right\| \leq C
$$

independent of $h$.

The pointwise convergence of $A_{h}$ to $A$ implies

$$
\left\|\left(A-A_{h}\right) \boldsymbol{J}\right\| \rightarrow 0 \quad \text { as } h \rightarrow 0,
$$

and similarly

$$
\left\|\left(A-A_{h}\right) A \boldsymbol{E}\right\| \rightarrow 0 \quad \text { as } h \rightarrow 0 .
$$

Hence we conclude the basic convergence of $\boldsymbol{E}_{h}$ to $\boldsymbol{E}$. 
The convergence of the source problem in the $H(\operatorname{curl} ; \Omega)$ then follows by using a Gårding inequality as in [22].

Corollary 5.2. Under the conditions of the previous theorem, if $\boldsymbol{E}$ satisfies (1.8) and $\boldsymbol{E}_{h}$ satisfies (1.14), then

$$
\left\|\boldsymbol{E}-\boldsymbol{E}_{h}\right\|_{H(\operatorname{curl} ; \Omega)} \rightarrow 0 \quad \text { as } h \rightarrow 0 .
$$

Proof. Let $e_{h}=\boldsymbol{E}-\boldsymbol{E}_{h}$, then using (1.8) and (1.14)

$$
\begin{aligned}
\left\|\nabla \times e_{h}\right\|^{2} & =\left(\left\|\nabla \times e_{h}\right\|^{2}-k^{2}\left\|e_{h}\right\|^{2}\right)+k^{2}\left\|e_{h}\right\|^{2} \\
& =\left[\left(\nabla \times\left(\boldsymbol{E}-\boldsymbol{E}_{h}\right), \nabla \times\left(\boldsymbol{E}-\boldsymbol{E}_{h}\right)\right)-k^{2}\left(\boldsymbol{E}-\boldsymbol{E}_{h}, \boldsymbol{E}-\boldsymbol{E}_{h}\right)\right]+k^{2}\left\|e_{h}\right\|^{2} \\
& =\left[\left(\nabla \times\left(\boldsymbol{E}-\boldsymbol{E}_{h}\right), \nabla \times\left(\boldsymbol{E}-\boldsymbol{\chi}_{h}\right)\right)-k^{2}\left(\boldsymbol{E}-\boldsymbol{E}_{h}, \boldsymbol{E}-\boldsymbol{\chi}_{h}\right)\right]+k^{2}\left\|e_{h}\right\|^{2}
\end{aligned}
$$

for any $\chi_{h} \in V_{h}$ (again we have used the fact that $V_{h}$ and $X_{h}$ differ only by gradients of functions in $S_{h}$ in the sense of (1.12)). Hence

$$
\left\|\boldsymbol{E}-\boldsymbol{E}_{h}\right\|_{H(\operatorname{curl} ; \Omega)} \leq C\left[\inf _{\chi_{h} \in V_{h}}\left\|\boldsymbol{E}-\chi_{h}\right\|_{H(\operatorname{curl} ; \Omega)}+\left\|\boldsymbol{E}-\boldsymbol{E}_{h}\right\|\right] .
$$

Convergence in the $\left(L^{2}(\Omega)\right)^{3}$ norm, and the density of $V_{h}$ in $H_{0}(\operatorname{curl} ; \Omega)$ completes the proof.

To establish rates of convergence, we need to assume a suitable regularity for the solution (and current in the case of the source problem).

Theorem 5.3. Suppose all the eigenfunctions $\phi \in E(\mu)$ are such that $\phi \in\left(H^{p}(\Omega)\right)^{3}$ and $\nabla \times \phi \in\left(H^{p}(\Omega)\right)^{3}$, then

$$
\left|\mu-\mu_{h, j}\right|=0\left(h^{2 p}\right) \quad 1 \leq j \leq m .
$$

Remark 8. For the cube, for example, this holds for any $p$.

Proof. Let $\phi \in E(\mu)$. Since $\nabla \cdot \phi=0$ we know that in (4.11), $p=0$. Hence using (4.11), Theorem 3.1 and the fact that $\phi$ is an eigenfunction

$$
\begin{aligned}
\left\|\left(A-A_{h}\right) \phi\right\|_{H(\operatorname{curl} ; \Omega)} & \leq C \inf _{\chi_{h} \in V_{h}}\left\|A \phi-\chi_{h}\right\|_{H(\operatorname{curl} ; \Omega)} \\
& \leq C h^{p}\left(\|A \phi\|_{p}+\|\nabla \times A \phi\|_{p}\right) \\
& =\frac{C h^{p}}{\mu}\left(\|\phi\|_{p}+\|\nabla \times \phi\|_{p}\right) .
\end{aligned}
$$

Since $E(\mu)$ is finite dimensional, this implies

$$
\left\|\left.\left(A-A_{h}\right)\right|_{E(\mu)}\right\|_{H(\operatorname{curl} ; \Omega)} \leq C_{\mu} h^{p},
$$

and we get the desired estimate.

Finally we can establish a convergence result for smooth solutions for the source problem.

Theorem 5.4. Suppose $A^{2} \boldsymbol{E} \in H^{p}(\operatorname{curl} ; \Omega)$ and $A \boldsymbol{J} \in H^{p}(\operatorname{curl} ; \Omega)$ where

$$
H^{p}(\operatorname{curl} ; \Omega)=\left\{\boldsymbol{u} \in\left(H^{p}(\Omega)\right)^{3} \mid \nabla \times \boldsymbol{u} \in\left(H^{p}(\Omega)\right)^{3}\right\} .
$$

Assuming the discrete compactness property, for sufficiently small $h$,

$$
\left\|\boldsymbol{E}-\boldsymbol{E}_{h}\right\| \leq C h^{p}\left(\left\|A^{2} \boldsymbol{E}\right\|_{H^{p}(\operatorname{curl} ; \Omega)}+\|A \boldsymbol{J}\|_{H^{p}(\operatorname{curl} ; \Omega)}\right) .
$$


If in addition $\boldsymbol{E} \in H^{p}(\operatorname{curl} ; \Omega)$, then

$$
\begin{aligned}
\left\|\boldsymbol{E}-\boldsymbol{E}_{h}\right\|_{H(\operatorname{curl} ; \Omega)} & \leq C h^{p}\left(\left\|A^{2} \boldsymbol{E}\right\|_{H^{p}(\operatorname{curl} ; \Omega)}+\|\mathbf{E}\|_{H^{p}(\operatorname{curl} ; \Omega)}\right. \\
& +\|A \boldsymbol{J}\|_{H^{p}(\operatorname{curl} ; \Omega)} .
\end{aligned}
$$

Proof. This is a straightforward application of Theorem 2.3. By Theorem 4.6 we obtain

$$
\begin{aligned}
\left\|\left(A-A_{h}\right) \boldsymbol{J}\right\| & \leq C h^{p}\left(\|A \boldsymbol{J}\|_{p}+\|\nabla \times A \boldsymbol{J}\|_{p}\right), \\
\left\|\left(A-A_{h}\right) A \boldsymbol{E}\right\| & \leq C h^{p}\left(\left\|A^{2} E\right\|_{p}+\left\|\nabla \times A^{2} \boldsymbol{E}\right\|_{p}\right) .
\end{aligned}
$$

To prove the second estimate we use (5.1) and Theorem 4.6 in addition to the $\left(L^{2}(\Omega)\right)^{3}$ estimate we have just proved.

\section{Conclusion}

We have given a unified treatment of the numerical analysis of the eigenvalue and source problems for Maxwell's equations using the theory of collectively compact operators. The key property in establishing the applicability of this theory is the discrete compactness property. We have shown, under the quasi-uniformity assumption, that the discrete compactness property is satisfied for two of the standard families of edge spaces hence extending Kikuchi's original result.

The weak points of our analysis are the need for the weak quasi-uniformity assumption since we would like to allow a geometrically graded mesh, and the $k$ dependence of the constants in our error estimates. This stems from our inability to estimate the term $\left\|\left(A-A_{h}\right) A_{h}\right\|$ in terms of $h$ (other than knowing $\left\|\left(A-A_{h}\right) A_{h}\right\| \rightarrow$ 0 as $h \rightarrow 0)$. Work on removing both these drawbacks of the theory is needed.

\section{ACKNOWLEDGMENTS AND DISCLAIMER}

Effort of Peter Monk was sponsored by the Air Force Office of Scientific Research, Air Force Materials Command, USAF, under grant number F49620-96-10039. Effort of Leszek Demkowicz was sponsored by the Air Force Office of Scientific Research, Air Force Materials Command, USAF, under grant number F49620-981-0255. The US Government is authorized to reproduce and distribute reprints for governmental purposes notwithstanding any copyright notation thereon. The views and conclusions contained herein are those of the authors and should not be interpreted as necessarily representing the official policies or endorsements, either expressed or implied, of the Air Force Office of Scientific Research or the US Government.

\section{REFERENCES}

[1] A. Alonso and A. Valli, An optimal domain decomposition preconditioner for low-frequency time-harmonic Maxwell equations. Math. Comp, 68 (1999), 607-631. MR 99i:78002

[2] P. Anselone, Collectively compact operator approximation theory, Prentice-Hall, 1971. MR 56:1753

[3] D. Boffi, F. Brezzi, And L. Gastaldi, On the convergence of eigenvalues for mixed formulations. Ann. Scuola Norm. Sup. Pisa Cl. Sci. (4) 25 (1997), 131-154. MR 99i:65121]

[4] - On the problem of spurious eigenvalues in the approximation of linear elliptic problems in mixed form. Math. Comp. to appear. CMP 98:17

[5] J. Bramble, J. Pasciak, And J. XU, The analysis of multigrid algorithms with nonnested or noninherited quadratic forms, Math. Comp. 56 (1991), pp. 1-34. MR 91h:65159

[6] M. Costabel, A remark on the regularity of solutions of Maxwell's equations on Lipschitz domains, Math. Meth. Appl. Sci., 12 (1990), pp. 365-368. MR 91c:35028 
[7] M. Costabel and M. Dauge, Singularities of electromagnetic fields in polyhedral domains, tech. report, IRMAR, Université de Rennes 1, France, 1997. Report available at http://www.maths . univ-rennes1.fr/ costabel/.

[8] L. Demkowicz, Asymptotic convergence in finite and boundary element methods. Part 1: Theoretical results, Comput. Math. Appl. 27 (1994), pp. 69-84. MR 95h:70653

[9] L. Demkowicz AND L. VARDAPETYAn, Modelling electromagnetic absorbtion/scattering problems using hp-adaptive finite elements, Comput. Methods Appl. Mech. Engrg., 152 (1998), pp. 103-124. MR 99b:78003

[10] V. GiRault, Incompressible finite element methods for Navier-Stokes equations with nonstandard boundary conditions in $\mathbb{R}^{3}$, Math. Comp., 51 (1988), pp. 55-74. MR 90e:65155

[11] _ Curl-conforming finite element methods for Navier-Stokes equations with nonstandard boundary conditions in $\mathbb{R}^{3}$, in The Navier-Stokes equations, Theory and Numerical Methods, Lecture Notes 1431, Springer, 1990, pp. 201-218. MR 91k:65143

[12] V. Girault and P. Raviart, Finite Element Methods for Navier-Stokes Equations, Springer-Verlag, New York, 1986. MR 83b:65122

[13] H. Ihlenburg And I. BabušKa, Finite element solution of the Helmholtz equation with high wavenumber Part I: The h-version of the FEM, Comput. Math. Appl., 30 (1995), pp. 9-37. MR 96j:65123

[14] P. Joly, C. Poirier, J.-E. Roberts, And P. Trouve, A new non-conforming finite element method for computation of electromagnetic guided waves, SIAM J. Numer. Anal., 33 (1996), 1494-1525. MR 97f:78035

[15] F. KIKUCHI, An isomorphic property of two Hilbert spaces appearing in electromagnetism: Analysis by the mixed formulation, Japan J. Appl. Math., 3 (1986), pp. 53-58. MR 88k:46028

[16] - Mixed and penalty formulations for finite element analysis of an eigenvalue problem in electromagnetism, Comput. Methods Appl. Mech. Eng., 64 (1987), pp. 509-521. MR 89g:78005

[17] - On a discrete compactness property for the Nedelec finite elements, J. Fac. Sci. Univ. Tokyo, Sect. 1A, Math, 36 (1989), pp. 479-490. MR 91h:65173

[18] R. KREss, Linear Integral Equations, Springer-Verlag, second ed., 1999. MR 90j:45001

[19] M. Křížek and P. NeittaAnmäKi, On the validity of Friedrichs' inequalities, Math. Scand., 54 (1984), pp. 17-26. MR 86b:35020

[20] R. LeIs, Initial Boundary Value Problems in Mathematical Physics, John Wiley, New York, 1986. MR 87h:35003

[21] V. Levillain, Eigenvalue appoximation by mixed methods for resonant inhomogenous cavities with metallic boundaries, Math. Comp., 58 (1992), pp. 11-20. MR 92f:65130

[22] P. Monk, A finite element method for approximating the time-harmonic Maxwell equations, Numer. Math., 63 (1992), pp. 243-261. MR 94b:65134

[23] J. NÉdÉlec, Mixed finite elements in $\mathbb{R}^{3}$, Numer. Math., 35 (1980), pp. 315-341. MR 81k:65125

[24] - Eléments finis mixtes incompressibles pour l'équation de Stokes dans $\mathbb{R}^{3}$, Numer. Math., 39 (1982), pp. 97-112. MR 83g:65111

[25] _ A new family of mixed finite elements in $\mathbb{R}^{3}$, Numer. Math., 50 (1986), pp. 57-81. MR 88e:65145

[26] J. Osborn, Spectral approximation for compact operators, Mathematics of Computation, 29 (1975), pp. 712-725. MR 52:3998

[27] W. Rachowicz and L. Demkowicz, A two-dimensional hp-adaptive finite element package for electromagnetics, Tech. Report 98-15, TICAM, University of Texas, 1998. submitted to Computer Methods in Applied Mechanics and Engineering.

[28] A. Schatz, An observation concerning Ritz-Galerkin methods with indefinite bilinear forms, Math. Comp., 28 (1974), pp. 959-962. MR 51:9526

[29] L. Vardapetyan and L. Demkowicz, hp-Adaptive finite elements in electromagnetics. Comput. Methods Appl. Mech. Engrg., 169 (1999), pp. 331-344. MR 99k:78004

Department of Mathematical Sciences, University of Delaware, Newark De 19716, USA

E-mail address: monk@math.udel.edu

TICAM, University of Texas at Austin, Austin TX 78712, USA

E-mail address: leszek@brahma.ticam.utexas.edu 